\title{
Turning points of nonlinear circuits
}

\author{
Ignacio García de la Vega ${ }^{1}$ and Ricardo Riaza ${ }^{2}$
}

\begin{abstract}
Bifurcation theory plays a key role in the qualitative analysis of dynamical systems. In nonlinear circuit theory, bifurcations of equilibria describe qualitative changes in the local phase portrait near an operating point, and are important from both an analytical and a numerical point of view. This work is focused on quadratic turning points, which, in certain circumstances, yield saddle-node bifurcations. Algebraic conditions guaranteeing the existence of this kind of points are well-known in the context of explicit ordinary differential equations (ODEs). We transfer these conditions to semiexplicit differential-algebraic equations (DAEs), in order to impose them to branch-oriented models of nonlinear circuits. This way, we obtain a description of the conditions characterizing these turning points in terms of the underlying circuit digraph and the devices' characteristics.
\end{abstract}

\section{Introduction}

The context of the present work is the study of bifurcation phenomena in nonlinear circuits. We have focused on quadratic turning points, which are related to certain local bifurcations in dynamical systems, in particular to the saddle-node bifurcation. With terminological abuse, we will often use the expression "turning point" to mean a "quadratic turning point". We are interested in the analysis of turning points in the equations governing nonlinear circuits, which have the structure of a semiexplicit DAE. Therefore, our first efforts are directed to adequate the classical conditions characterizing turning points in ODEs to a semiexplicit index-one DAE context (Section 2). Afterwards, in Section 3 , we will analyze these reformulated

\footnotetext{
12 Depto. de Matemática Aplicada a las Tecnologías de la Información y las Comunicaciones Escuela Técnica Superior de Ingenieros de Telecomunicación Universidad Politécnica de Madrid

Ciudad Universitaria s/n. 28040 - Madrid, Spain

e-mail: ignacio.garciadelavega@gmail.com,ricardo.riaza@upm.es
}

This is the authors' version of the paper published in the proceedings of the Scientific Computing in Electrical Enginnering 2014 conference, Mathematics in Industry 23 (2016) pp. 21-29, Springer. The final publication is available at Springer, cf. http://dx.doi.org/10.1007/978-3-319-30399-4_3 
conditions in terms of the circuit topology and the devices' characteristics. Finally, Section 4 briefly compiles some concluding remarks.

Turning points in explicit ODEs. Let us recall the algebraic conditions defining quadratic turning points in ODEs. Consider the ordinary differential equation

$$
x^{\prime}=f(x, \mu),
$$

with $x \in \mathbb{R}^{n}$, and $f$ sufficiently smooth and depending on a parameter $\mu \in \mathbb{R}$. Provided that $f\left(x^{*}, \mu^{*}\right)=0,\left(x^{*}, \mu^{*}\right)$ is called a quadratic turning point of (1) if the conditions 1-3 below are satisfied [4].

1. $\mathrm{rk} f_{x}\left(x^{*}, \mu^{*}\right)=n-1$;

2. $w^{T} f_{\mu}\left(x^{*}, \mu^{*}\right) \neq 0$;

3. $w^{T} f_{x x}\left(x^{*}, \mu^{*}\right)(v, v) \neq 0$.

Here $v$ (resp. $w$ ) denotes a right (resp. left) eigenvector of the zero eigenvalue of the matrix of partial derivatives $f_{x}\left(x^{*}, \mu^{*}\right)$. Such turning points are important e.g. in numerical continuation theory [1]. If, additionally,

4. the algebraic multiplicity of the null eigenvalue of $f_{x}\left(x^{*}, \mu^{*}\right)$ is one; and

5. the remaining eigenvalues of $f_{x}\left(x^{*}, \mu^{*}\right)$ have non-zero real parts,

then $\left(x^{*}, \mu^{*}\right)$ is called a saddle-node bifurcation point, because the system undergoes a saddle-node bifurcation as $\mu$ crosses $\mu^{*}[5,7,10]$. Near $\left(x^{*}, \mu^{*}\right)$ we will observe that when $\mu<\mu^{*}$ (resp. when $\mu>\mu^{*}$ ) there are no equilibria, whereas for $\mu>\mu^{*}$ (resp. $\mu<\mu^{*}$ ) there are two hyperbolic equilibrium points. These two equilibria differ in the sign of one real eigenvalue, being in particular a saddle and a node when $x \in \mathbb{R}^{2}$.

\section{Turning points in semiexplicit DAEs}

Our purpose is to characterize the existence of turning points and saddle-node bifurcations in electrical circuit models and, specifically, in branch-oriented models. These models have the structure of a semiexplicit DAE [3,8], that is,

$$
\begin{aligned}
y^{\prime} & =h(y, z, \mu) \\
0 & =g(y, z, \mu),
\end{aligned}
$$

where $y \in \mathbb{R}^{r}, z \in \mathbb{R}^{p}, \mu \in \mathbb{R}$, and $h$ and $g$ are sufficiently smooth. We will group together $y$ and $z$ into a single variable $x=(y, z) \in \mathbb{R}^{n}$, with $n=r+p$. For later use let us also define the matrices

$$
M=\left(\begin{array}{ll}
h_{y} & h_{z} \\
g_{y} & g_{z}
\end{array}\right), \tilde{M}=\left(\begin{array}{c}
M \\
(\operatorname{det} M)_{x}
\end{array}\right) .
$$


Specifically, we will work in a local index-one context [3],8]; this means that the matrix of partial derivatives $g_{z}\left(y^{*}, z^{*}, \mu^{*}\right)$ is non-singular. By the implicit function theorem this implies that there is a local map $\psi(y, \mu)$ such that $0=g(y, z, \mu)$ if and only if $z=\psi(y, \mu)$, with $\psi_{y}=-\left(g_{z}\right)^{-1} g_{y}$. This, together with 2ab), enables one to express the local dynamics of the DAE (2) in terms of the reduced ODE

$$
y^{\prime}=\eta(y, \mu)
$$

with $\eta(y, \mu)=h(y, \psi(y, \mu), \mu)$. In turn, this makes it possible to define an equilibrium $\left(y^{*}, z^{*}, \mu^{*}\right)$ of the semiexplicit index-one DAE (2) as a (quadratic) turning point (resp. a saddle-node bifurcation point) if the reduction (4) satisfies the conditions 1-3 (resp. 1-5) stated in Section1.

Theorem 1 provides conditions for system (2) to have a turning point. Additional conditions for the existence of a saddle-node point will be formulated in terms of the reduction (4); this point of view will be exploited in Section 3

Theorem 1. Consider the semiexplicit DAE (2) and assume there exists a point $\left(x^{*}, \mu^{*}\right)$ such that $h\left(x^{*}, \mu^{*}\right)=0$ and $g\left(x^{*}, \mu^{*}\right)=0$, with $g_{z}\left(x^{*}, \mu^{*}\right)$ non-singular. Then $\left(x^{*}, \mu^{*}\right)$ is a quadratic turning point if the following conditions are satisfied:

1. $\operatorname{rk} M\left(x^{*}, \mu^{*}\right)=n-1$;

2. $\left(\begin{array}{c}h_{\mu} \\ g_{\mu}\end{array}\right)\left(x^{*}, \mu^{*}\right) \notin \operatorname{im} M\left(x^{*}, \mu^{*}\right)$;

3. $\operatorname{rk} \tilde{M}\left(x^{*}, \mu^{*}\right)=n$.

Proof. Write $x^{*}=\left(y^{*}, z^{*}\right)$ and note that $\left(y^{*}, \mu^{*}\right)$ is an equilibrium point of [4], because $\eta\left(y^{*}, \mu^{*}\right)=h\left(y^{*}, z^{*}, \mu^{*}\right)=0$. We check below that conditions 1-3 in Section 1 hold for the reduction (4) at $\left(y^{*}, \mu^{*}\right)$.

1. If we compute $\eta_{y}$ in terms of the maps $h$ and $g$, we obtain

$$
\eta_{y}=\left(\begin{array}{ll}
h_{y} & h_{z}
\end{array}\right)\left(\begin{array}{c}
I \\
-\left(g_{z}\right)^{-1} g_{y}
\end{array}\right)=h_{y}-h_{z}\left(g_{z}\right)^{-1} g_{y},
$$

which is the Schur complement of $g_{z}$ in $M$ [6]. The corank of a matrix and the corank of its Schur reduction are equal, therefore $\operatorname{rk} M\left(x^{*}, \mu^{*}\right)=n-1$ implies $\mathrm{rk} \eta_{y}\left(y^{*}, \mu^{*}\right)=r-1$.

2. The second condition is $w^{T} \eta_{\mu}\left(y^{*}, \mu^{*}\right) \neq 0$, where $w$ is an eigenvector associated to the zero eigenvalue of the matrix $A^{T}$ with $A=\eta_{y}\left(y^{*}, \mu^{*}\right)$; note that $w^{T} A=0 \Leftrightarrow w^{T} \perp \operatorname{im~A}$. Therefore, $w^{T} \eta_{\mu}\left(y^{*}, \mu^{*}\right) \neq 0 \Leftrightarrow \eta_{\mu}\left(y^{*}, \mu^{*}\right) \notin \operatorname{im} A$, that is, $\left(h_{\mu}-h_{z} g_{z}^{-1} g_{\mu}\right)\left(x^{*}, \mu^{*}\right) \notin \operatorname{im}\left(h_{y}-h_{z}\left(g_{z}\right)^{-1} g_{y}\right)\left(x^{*}, \mu^{*}\right)$ which is equivalent to

$$
\left(\begin{array}{l}
h_{\mu} \\
g_{\mu}
\end{array}\right)\left(x^{*}, \mu^{*}\right) \notin \operatorname{im}\left(\begin{array}{ll}
h_{y} & h_{z} \\
g_{y} & g_{z}
\end{array}\right)\left(\mathrm{x}^{*}, \mu^{*}\right) .
$$

3. Equation $w^{T} \eta_{y y}\left(y^{*}, \mu^{*}\right)(v, v) \neq 0$ can be recast as $\eta_{y y}\left(y^{*}, \mu^{*}\right)(v, v) \notin \operatorname{im} \eta_{y}\left(y^{*}, \mu^{*}\right)$. The fact that for a $C^{2}$ map $f: \mathbb{R}^{n} \rightarrow \mathbb{R}^{n}$ satisfying cork $f^{\prime}\left(x^{*}\right)=1$ we have $\left(\operatorname{det} f^{\prime}(x)\right)^{\prime} v \neq 0 \Leftrightarrow f^{\prime \prime}(x)(v, v) \notin \operatorname{im} f^{\prime}(x)$, where $v$ is a non-null vector belonging to 
$\operatorname{ker} f^{\prime}(x)$, allows us to transform this condition into $\left(\operatorname{det}\left(\eta_{y}\right)\right)_{y}\left(y^{*}, \mu^{*}\right) \nu \neq 0$, where $v \in \operatorname{ker} \eta_{y}\left(y^{*}, \mu^{*}\right)$. Additionally, because $\eta_{y}$ is the Schur complement of $g_{z}$ in $M$, we have $\operatorname{det} \eta_{y}=\operatorname{det}\left(g_{z}^{-1}\right) \operatorname{det} M$, and then

$$
\left(\operatorname{det}\left(\eta_{y}\right)\right)_{y}=\left(\operatorname{det}\left(g_{z}^{-1}\right)\right)_{x} \operatorname{det} M\left(\begin{array}{c}
I \\
-\left(g_{z}\right)^{-1} g_{y}
\end{array}\right)+\operatorname{det}\left(g_{z}^{-1}\right)(\operatorname{det} M)_{x}\left(\begin{array}{c}
I \\
-\left(g_{z}\right)^{-1} g_{y}
\end{array}\right) .
$$

Condition 1 states that $\operatorname{rk} M\left(x^{*}, \mu^{*}\right)=n-1$, thus $\operatorname{det}\left(M\left(x^{*}, \mu^{*}\right)\right)=0$. Additionally, det $\left(g_{z}^{-1}\right)\left(x^{*}, \mu^{*}\right) \neq 0$; therefore condition 3 is satisfied if and only if

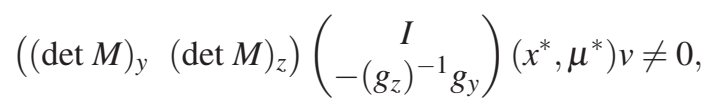

for some (hence any) non-vanishing vector $v$ belonging to $\operatorname{ker} \eta_{y}\left(y^{*}, \mu^{*}\right)$. Because of the identity $\operatorname{ker} \eta_{y}\left(y^{*}, \mu^{*}\right)=\operatorname{ker}\left(h_{y}-h_{z}\left(g_{z}\right)^{-1} g_{y}\right)\left(x^{*}, \mu^{*}\right)$, condition 3 is then equivalent to the requirement that the system

$$
\begin{aligned}
\left(h_{y}-h_{z}\left(g_{z}\right)^{-1} g_{y}\right)\left(x^{*}, \mu^{*}\right) v & =0 \\
\left((\operatorname{det} M)_{y}-(\operatorname{det} M)_{z} g_{z}^{-1} g_{y}\right)\left(x^{*}, \mu^{*}\right) v & =0
\end{aligned}
$$

only possesses the trivial solution. Equivalently, the matrix of coefficients of (5),

$$
M_{1}=\left(\begin{array}{c}
h_{y}-h_{z}\left(g_{z}\right)^{-1} g_{y} \\
\operatorname{det} M_{y}-\operatorname{det} M_{z} g_{z}^{-1} g_{y}
\end{array}\right)\left(x^{*}, \mu^{*}\right),
$$

must have maximum column rank. But $M_{1}$ is the Schur complement of $g_{z}$ in the matrix $\tilde{M}\left(x^{*}, \mu^{*}\right)$ arising in the statement of condition 3 of Theorem 1, hence, the maximum column rank condition on $M_{1}$ is transferred to $\tilde{M}\left(x^{*}, \mu^{*}\right)$. This means that condition 3 in Section 1 holds for (4) at $\left(y^{*}, \mu^{*}\right)$ and the proof is complete.

\section{Nonlinear circuits exhibiting turning points}

In this section, we characterize the existence of turning points and saddle-node bifurcations for nonlinear circuits, under certain restrictions to be specified later. For this purpose, we use branch-oriented circuit models [8] defined by:

$$
\begin{aligned}
C\left(v_{c}\right) v_{c}^{\prime} & =i_{c} \\
L\left(i_{l}\right) i_{l}^{\prime} & =v_{l} \\
0 & =B_{c} v_{c}+B_{l} v_{l}+B_{g} v_{g}+B_{n} v_{n}+B_{j} v_{j}+B_{v} V \\
0 & =Q_{c} i_{c}+Q_{l} i_{l}+Q_{g} \gamma_{1}\left(v_{g}\right)+Q_{n} \gamma_{2}\left(v_{n}\right)+Q_{j} \mu+Q_{v} i_{v},
\end{aligned}
$$

where we denote the branch voltages by $v$, the currents by $i$, and use the subscripts $c, l, g, n, j$ and $v$ to denote capacitors, inductors, passive resistors, non-passive resis- 
tors, current sources and voltage sources, respectively. All devices may be nonlinear, often without explicit mention. We assume that there exists only one non-passive resistor and a unique DC current source, whose current $i_{j}=\mu$ is the parameter of the system. The reader may think of a tunnel diode as an example of a (locally) nonpassive resistor. We also assume that there exists an equilibrium point that we will denote by $\left(x^{*}, \mu^{*}\right)=\left(v_{c}^{*}, i_{l}^{*}, i_{c}^{*}, v_{l}^{*}, v_{g}^{*}, v_{n}^{*}, v_{j}^{*}, i_{v}^{*}, \mu^{*}\right)$. The incremental capacitance and inductance matrices, $C$ and $L$, are both non-singular at $\left(x^{*}, \mu^{*}\right)$ and, finally, $V$ is the vector of voltages in the DC voltage sources.

System (6) has the semiexplicit DAE structure displayed in (2) with $y=\left(v_{c}, i_{l}\right)$ and $z=\left(i_{c}, v_{l}, v_{g}, v_{n}, v_{j}, i_{v}\right)$. Note that equations $6 \mathrm{ab}$ and $6 \mathrm{~b}$ stand for the constitutive relations of capacitors and inductors, whereas equations (6c) and (6d) are the expression of Kirchhoff laws. In (6d) we have eliminated the resistors currents using the constitutive relations $\gamma_{1}$ and $\gamma_{2}$. In the formulation of Kirchhoff laws we have made use of the so-called loop and cutset matrices $B, Q$, which are well-known in digraph theory and whose main properties are compiled in Lemma 1 [2, 9].

Lemma 1. The loop and cutset matrices $B, Q$ of a digraph verify the following.

1. $B_{K}\left(\right.$ resp. $\left.Q_{K}\right)$ has full column rank if and only if the branches specified by $K$ do not contain any cutset (resp. loop).

2. The loop and cutset spaces are orthogonal to each other, that is, if columns of $Q$ and $B$ are arranged in the same order, then $Q B^{T}=0$.

3. Suppose the branches of a given digraph are split in four disjoint sets $K_{1}, K_{2}, K_{3}$ and $K_{4}$, and denote by $B_{i}$ and $Q_{i}$ the submatrices of the loop and cutset matrices defined by $K_{i}$; assume additionally that $P$ is a positive definite matrix. Then

$$
\operatorname{ker}\left(\begin{array}{ccc}
B_{1} & 0 & B_{3} \\
0 & Q_{2} & Q_{3} P
\end{array}\right)=\operatorname{ker} B_{1} \times \operatorname{ker} Q_{2} \times\{0\} .
$$

These properties allow us to prove Theorem 2, which characterizes turning points and saddle-node bifurcations for the circuit model (6). By a $K$-loop (resp. $K$-cutset) we mean a loop (resp. cutset) defined only by elements of $K$; this way, for instance a JCN-cutset is a cutset defined only by current sources, capacitors and/or non-passive resistors. JLN-cutsets, VC-loops, etc. are defined analogously.

Theorem 2. In the setting defined above, assume that $\gamma_{2}^{\prime}\left(v_{n}^{*}\right)=0, \gamma_{2}^{\prime \prime}\left(v_{n}^{*}\right) \neq 0$ at the equilibrium point $\left(x^{*}, \mu^{*}\right)$. This equilibrium is then a turning point of (6) if

- there is a unique JCN-cutset, which includes the current source, the non-passive resistor and at least one capacitor; and

- there are no JLN-cutsets, VC-loops or JVL-loops.

If, additionally, L and C are symmetric positive definite and there are no VCL-loops, then the turning point yields a saddle-node bifurcation.

Proof. The matrices $g_{z}\left(x^{*}, \mu^{*}\right)$ and $M\left(x^{*}, \mu^{*}\right)$ read for system (6) as: 
$g_{z}\left(x^{*}, \mu^{*}\right)=\left(\begin{array}{cccccc}0 & B_{l} & B_{g} & B_{n} & B_{j} & 0 \\ Q_{c} & 0 & Q_{g} G & 0 & 0 & Q_{v}\end{array}\right), M\left(x^{*}, \mu^{*}\right)=\left(\begin{array}{cccccccc}0 & 0 & C^{-1} & 0 & 0 & 0 & 0 & 0 \\ 0 & 0 & 0 & L^{-1} & 0 & 0 & 0 & 0 \\ B_{c} & 0 & 0 & B_{l} & B_{g} & B_{n} & B_{j} & 0 \\ 0 & Q_{l} & Q_{c} & 0 & Q_{g} G & 0 & 0 & Q_{v}\end{array}\right)$,

where $G=\gamma_{1}^{\prime}\left(v_{g}^{*}\right)$ is the incremental conductance matrix of passive resistors, which is positive definite. In light of item 3 in Lemma1 non-trivial entries in $\operatorname{ker} g_{z}\left(x^{*}, \mu^{*}\right)$ must come either from $\operatorname{ker}\left(B_{l} B_{n} B_{j}\right)$ or from $\operatorname{ker}\left(Q_{c} Q_{v}\right)$. Since there are neither JLN-cutsets nor VC-loops, we conclude that $g_{z}\left(x^{*}, \mu^{*}\right)$ is non-singular.

1. The non-singularity of $C, L$ allows us to study the rank of the matrix $M\left(x^{*}, \mu^{*}\right)$ in terms of

$$
\left(\begin{array}{cccccc}
B_{c} & 0 & B_{g} & B_{n} & B_{j} & 0 \\
0 & Q_{l} & Q_{g} G & 0 & 0 & Q_{v}
\end{array}\right) .
$$

By applying item 3 of Lemma1, non-zero entries of $\operatorname{ker} M\left(x^{*}, \mu^{*}\right)$ must come either from $\operatorname{ker}\left(B_{c} B_{n} B_{j}\right)$ or from $\operatorname{ker}\left(Q_{l} Q_{v}\right)$. Since there is a unique JCN-cutset and no JVL-loops, we have null $\left(B_{c} B_{n} B_{j}\right)=1$, where null stands for the nullity, that is, the dimension of the kernel, null $\left(Q_{l} Q_{v}\right)=0$ and therefore null $M\left(x^{*}, \mu^{*}\right)=1$, that is, $\operatorname{rk} M\left(x^{*}, \mu^{*}\right)=n-1$, which is condition 1 in Theorem 1 .

2. The second condition in Theorem 1 may be restated as null $M\left(x^{*}, \mu^{*}\right)=\operatorname{null} \hat{M}\left(x^{*}, \mu^{*}\right)$, with

$$
\hat{M}\left(x^{*}, \mu^{*}\right)=\left(\begin{array}{ccccccccc}
0 & 0 & C^{-1} & 0 & 0 & 0 & 0 & 0 & 0 \\
0 & 0 & 0 & L^{-1} & 0 & 0 & 0 & 0 & 0 \\
B_{c} & 0 & 0 & B_{l} & B_{g} & B_{n} & B_{j} & 0 & 0 \\
0 & Q_{l} & Q_{c} & 0 & Q_{g} G & 0 & 0 & Q_{\nu} & Q_{j}
\end{array}\right) .
$$

Proceeding as above, we observe that non-trivial entries in $\operatorname{ker} \hat{M}\left(x^{*}, \mu^{*}\right)$ must be due to those in $\operatorname{ker}\left(B_{c} B_{n} B_{j}\right)$ or in $\operatorname{ker}\left(Q_{l} Q_{v} Q_{j}\right)$. The absence of JVL-loops implies null $\hat{M}\left(x^{*}, \mu^{*}\right)=\operatorname{null}\left(B_{c} B_{n} B_{j}\right)$ and therefore null $M=\operatorname{null} \hat{M}$.

3. The third condition in Theorem 1 says that the matrix $\tilde{M}$ (cf. (3)) has full column rank or, equivalently, $\operatorname{rk} \tilde{M}=n$. Provided that null $M=1$, requiring $\tilde{M}$ to have full column rank is equivalent to $(\operatorname{det} M)_{x} v \neq 0$, where $v$ is any vector that spans $\operatorname{ker} M$. For any point $\hat{x}=\left(v_{c}, i_{l}, i_{c}, v_{l}, v_{g}, v_{n}^{*}, v_{j}, i_{v}\right), M(\hat{x}, \mu)$ is a singular matrix because $\gamma_{2}^{\prime}\left(v_{n}^{*}\right)=0$. Thus, $(\operatorname{det} M)_{x}=\left(\begin{array}{llllllll}0 & 0 & 0 & 0 & 0 & a & 0 & 0\end{array}\right)$ and $a \neq 0$ because $\gamma_{2}^{\prime \prime}\left(v_{n}^{*}\right) \neq 0$.

The absence of VL-loops and the existence of a JCN-cutset imply that vectors belonging to $\operatorname{ker} M$ have the form of $v$ where $v^{T}=\left(v_{1}, 0,0,0,0, v_{6}, v_{7}, 0\right)$. Additionally, the fact that there are no JC-cutsets implies $v_{6} \neq 0$; it follows that the multiplication of $(\operatorname{det} M)_{x}$ by vectors of $\operatorname{ker} M$ does not vanish.

4. To complete the proof it remains to show that the absence of VCL-loops leads to a saddle-node bifurcation. To do this we make use of conditions 4 and 5 in Section 1.

In order to prove that the zero eigenvalue is simple, we will show that the intersection of the kernel and the image of $\eta_{y}=\left(h_{y}-h_{z}\left(g_{z}\right)^{-1} g_{y}\right)$ at $\left(x^{*}, \mu^{*}\right)$ only contains the null vector. First, a vector $u$ belongs to im $\eta_{y}$ if and only if $\hat{u}$ belongs to $\operatorname{im} M$, with $\hat{u}^{T}=\left(u^{T} 0\right)$, that is, if and only if there exists a vector $v$ satisfying 
Turning points of nonlinear circuits

$$
\begin{aligned}
u_{1} & =C^{-1} v_{3} \\
u_{2} & =L^{-1} v_{4} \\
0 & =B_{c} v_{1}+B_{l} v_{4}+B_{g} v_{5}+B_{n} v_{6}+B_{j} v_{7} \\
0 & =Q_{l} v_{2}+Q_{c} v_{3}+Q_{g} G v_{5}+Q_{v} v_{8} .
\end{aligned}
$$

On the other hand, a vector $u$ belongs to ker $\eta_{y}$ if and only if

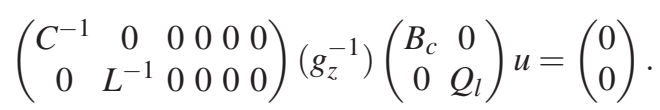

In order to satisfy this relation there must be a vector $y$ such that

$$
\left(g_{z}^{-1}\right)\left(\begin{array}{l}
B_{c} u_{1} \\
Q_{l} u_{2}
\end{array}\right)=\left(\begin{array}{c}
0 \\
0 \\
y_{1} \\
y_{2} \\
y_{3} \\
y_{4}
\end{array}\right) \Leftrightarrow\left(\begin{array}{l}
B_{c} u_{1} \\
Q_{l} u_{2}
\end{array}\right)=g_{z}\left(\begin{array}{c}
0 \\
0 \\
y_{1} \\
y_{2} \\
y_{3} \\
y_{4}
\end{array}\right),
$$

that is,

$$
\begin{aligned}
& B_{c} u_{1}=B_{g} y_{1}+B_{n} y_{2}+B_{j} y_{3} \\
& Q_{l} u_{2}=Q_{g} G y_{1}+Q_{v} y_{4} .
\end{aligned}
$$

Using the orthogonality of the cutset and loop spaces, namely, the fact that $\operatorname{ker} B$ and $\operatorname{ker} Q$ are orthogonal to one another (cf. [2]), it is not difficult to obtain from (8) the relation $y_{1}^{T} G y_{1}=0 ; y_{1}$ must then vanish because $G$ is positive definite. Making use of $(7 \mathrm{a})$ and $(7 \mathrm{~b})$, equations (8a) and (8b) then read as

$$
\begin{aligned}
& 0=B_{c} C^{-1} v_{3}-B_{n} y_{2}-B_{j} y_{3} \\
& 0=Q_{l} L^{-1} v_{4}-Q_{v} y_{4} .
\end{aligned}
$$

Therefore if $u \in \operatorname{ker} \eta_{y} \cap \operatorname{im} \eta_{y}$, then (7c), (7d) and (9) must hold. Applying the aforementioned orthogonality property to (7c) and (9b) we obtain that $v_{4}^{T} L^{-1} v_{4}=0$ and from equations (7d) and 9a , $v_{3}^{T} C^{-1} v_{3}=0$. Altogether this yields $u=0$.

5. It remains to be proved that if there are no VCL-loops then $\eta_{y}\left(x^{*}, \mu^{*}\right)$ has no purely imaginary eigenvalues. A complex number $\lambda$ is an eigenvalue of $\eta_{y}\left(x^{*}, \mu^{*}\right)$ if and only if

$$
\lambda\left(\begin{array}{ll}
I & 0 \\
0 & 0
\end{array}\right)-\left(\begin{array}{ll}
h_{y} & h_{z} \\
g_{y} & g_{z}
\end{array}\right)\left(x^{*}, \mu^{*}\right)
$$

is singular or, equivalently, there exists non-trivial solutions to

$$
\begin{aligned}
& 0=\lambda^{-1} B_{c} C^{-1} i_{c}+\lambda B_{l} L i_{l}+B_{g} v_{g}+B_{n} v_{n}+B_{i} v_{i} \\
& 0=Q_{c} i_{c}+Q_{l} i_{l}+Q_{g} G v_{g}+Q_{v} i_{v} .
\end{aligned}
$$


The orthogonality of the cutset and cycle spaces implies that if $Q p=0$ and $B q=0$ then $p^{T} q=0$. Applying this result to the conjugate of $10 \mathrm{~b}$ in conjunction with 10a, we obtain

$$
0=\lambda^{-1} i_{c}^{*} C^{-1} i_{c}+\lambda i_{l}^{*} L i_{l}+v_{g}^{*} G v_{g},
$$

where ${ }^{*}$ stands for the Hermitian (conjugate transpose). If we take the sum of (11) and its Hermitian, we obtain:

$$
0=2 \operatorname{Re}\left(\lambda^{-1}\right) i_{c}^{*} C^{-1} i_{c}+2 \operatorname{Re}(\lambda) i_{l}^{*} L i_{l}+v_{g}^{*}\left(G+G^{T}\right) v_{g} .
$$

For purely imaginary eigenvalues, $\operatorname{Re}\left(\lambda^{-1}\right)=\operatorname{Re}(\lambda)=0$ and therefore we must have $v_{g}=0$ for (12) to hold. System (10) can be then simplified to:

$$
\begin{aligned}
& 0=\lambda^{-1} B_{c} C^{-1} i_{c}+\lambda B_{l} L i_{l}+B_{n} v_{n}+B_{i} v_{i} \\
& 0=Q_{c} i_{c}+Q_{l} i_{l}+Q_{v} i_{v} .
\end{aligned}
$$

Since there are no VCL-loops, $\left(Q_{c} Q_{l} Q_{v}\right)$ has full column rank and, consequently, $i_{c}=i_{l}=i_{v}=0$ must hold to satisfy $113 \mathrm{~b}$. The absence of JLN-cutsets then yields $v_{n}=v_{i}=0$ from (13a). This means that (10) only has the trivial solution, and this rules out purely imaginary eigenvalues. The proof of Theorem 2 is then complete.

\section{Concluding remarks}

We have performed a circuit-theoretic analysis of the existence of turning points and saddle-node bifurcations in nonlinear circuits. The analysis of these phenomena in broader contexts, including e.g. other non-passive devices, higher-index configurations or parameters with other roles, as well as the study of other related bifurcations in similar terms, are in the scope of future research.

Acknowledgements Research supported by Project MTM2010-15102 of Ministerio de Ciencia e Innovación, Spain.

\section{References}

1. E. L. Allgower and K. Georg, Introduction to Numerical Continuation Methods. SIAM, Philadelphia, 2003.

2. B. Bollobás, Modern Graph Theory. Springer-Verlag, New York, 1998.

3. K. E. Brenan, S. L. Campbell and L. R. Petzold. Numerical Solution of Initial-Value Problems in Differential Algebraic Equations. SIAM, Philadelphia, 1996.

4. W. J. F. Govaerts. Numerical Methods for Bifurcations of Dynamical Equilibria. SIAM, Philadelphia, 2000.

5. J. Guckenheimer and P. Holmes. Nonlinear Oscillations, Dynamical Systems, and Bifurcations of Vector Fields. Springer-Verlag, New York, 1983.

6. R. A. Horn and Ch. R. Johnson, Matrix Analysis, Cambridge University Press, 1985. 
7. L. Perko. Differential Equations and Dynamical Systems. Springer-Verlag, New York, 1991.

8. R. Riaza, Differential-Algebraic Systems. World Scientific, Singapore, 2008.

9. R. Riaza, Manifolds of equilibria and bifurcations without parameters in memristive circuits. SIAM J. Applied Mathematics 72 (2012) 877-896.

10. J. Sotomayor. Generic bifurcations of dynamical systems. In Dynamical Systems, M. M. Peixoto (ed.). Academic Press, New York, 561-582, 1973. 